\title{
Sintomatologia in emodialisi e possibili cause
}

\author{
G. Virga ${ }^{1}$, S. Mastrosimone ${ }^{1}$, G. Amici ${ }^{2}$, G. Munaretto ${ }^{1}$, F. Gastaldon ${ }^{1}$, A. Bonadonna ${ }^{1}$ \\ ${ }^{1}$ Dipartimento di Nefro-Urologia, Ospedale Provinciale, Camposampiero (Padova) \\ ${ }^{2}$ Divisione di Nefrologia e Dialisi, Ospedale Regionale - Treviso
}

a sindrome uremica è l'insieme dei segni e sintomi indotti dalle alterazioni metaboliche dell'insufficienza renale e dalle complicanze legate al suo trattamento. La qualità della vita dei pazienti $(\mathrm{pz})$ in emodialisi (HD) è fortemente condizionata da tale sintomatologia che è stata studiata soprattutto nell'ambito della seduta dialitica (1, 2). Il miglioramento della loro qualità della vita è uno degli obiettivi prioritari della terapia sostitutiva insieme all'allungamento della loro sopravvivenza. Lo scopo del nostro lavoro è quello di valutare, nei $\mathrm{pz}$ in $\mathrm{HD}$, la presenza e frequenza a domicilio dei più comuni sintomi uremici $e$, al fine di un'eventuale prevenzione e trattamento, studiare le loro relazioni con un set il più possibile ampio di parametri bioumorali e demografici.

\section{Materiali e Metodi}

\section{Pazienti}

Tutti i pz del nostro Centro $(n=76)$ sono stati arruolati per lo studio trasversale e, successivamente, tre di essi sono stati esclusi perché giudicati inattendibili a causa di schizofrenia $(n=1)$, oligofrenia $(n=1)$ e psi-

\begin{tabular}{|c|c|}
\hline Maschi & $47(61.8 \%)$ \\
\hline Femmine & $29(38.2 \%)$ \\
\hline Età anagrafica (anni) & $67.0(57.6-72.1)$ \\
\hline Età dialitica (mesi) & $37.2(19.5-90.4)$ \\
\hline $\mathrm{Hb}(\mathrm{g} / \mathrm{dL})$ & $10.2 \pm 1.3$ \\
\hline Albumina $(\mathrm{g} / \mathrm{dL})$ & $4.3(4.1-4.5)$ \\
\hline $\mathrm{Na}(\mathrm{mEq} / \mathrm{L})$ & $137.5 \pm 2.9$ \\
\hline $\mathrm{K}(\mathrm{mEq} / \mathrm{L})$ & $5.5 \pm 0.8$ \\
\hline $\mathrm{BUN}(\mathrm{mg} / \mathrm{dL})$ & $73.1 \pm 19.2$ \\
\hline Creatinina (mg/dL) & $9.9(8.5-11.5)$ \\
\hline Glucosio (mg/dL) & $106(91-128)$ \\
\hline Calcio $(\mathrm{mg} / \mathrm{dL})$ & $9.9 \pm 0.7$ \\
\hline Fosforo (mg/dL) & $4.8 \pm 1.6$ \\
\hline PTHi (ng/mL) & $93(41-291)$ \\
\hline $\mathrm{pH}$ & $7.395 \pm 0.042$ \\
\hline $\mathrm{pCO}_{2}(\mathrm{mmHg})$ & $37.0 \pm 3.9$ \\
\hline $\mathrm{pO}_{2}(\mathrm{mmHg})$ & $85.7 \pm 12.7$ \\
\hline $\mathrm{HCO}_{3}(\mathrm{mmHg})$ & $22.3 \pm 3.3$ \\
\hline $\mathrm{BE}(\mathrm{mEq} / \mathrm{L})$ & $-1.8 \pm 3.3$ \\
\hline Osmolalità (mOsm/kg) & $307.3 \pm 9.7$ \\
\hline $\mathrm{Kt} / \mathrm{V}$ & $1.29 \pm 0.23$ \\
\hline Pressione sistolica $(\mathrm{mmHg})$ & $140 \pm 22$ \\
\hline Pressione diastolica $(\mathrm{mmHg})$ & $79 \pm 13$ \\
\hline Peso corporeo $(\mathrm{kg})$ & $63.0 \pm 11.2$ \\
\hline
\end{tabular}


TABELLA II - PRESENZA E FREQUENZA DEI 14 SINTOMI STUDIATI IN EMODIALISI SECONDO GLI SCORE 2 ("TUTTI I GIORNI"), 1 ("NON TUTTI I GIORNI") E 0 ("MAI")

\begin{tabular}{llll}
\hline & score 2 & score 1 & score 0 \\
\hline 1) Sete & $49.3 \%$ & $35.6 \%$ & $15.1 \%$ \\
2) Astenia & $26.0 \%$ & $63.0 \%$ & $11.0 \%$ \\
3) Artralgie & $21.9 \%$ & $27.4 \%$ & $50.7 \%$ \\
4) Insonnia & $20.5 \%$ & $37.0 \%$ & $42.5 \%$ \\
5) Ipersonnia & $15.1 \%$ & $38.3 \%$ & $46.6 \%$ \\
6) Prurito & $9.6 \%$ & $57.5 \%$ & $32.9 \%$ \\
7) Anoressia & $8.2 \%$ & $34.3 \%$ & $57.5 \%$ \\
8) RLS & $8.2 \%$ & $19.2 \%$ & $72.6 \%$ \\
9) Tremori & $8.2 \%$ & $16.5 \%$ & $75.3 \%$ \\
10) Dispnea & $4.1 \%$ & $38.4 \%$ & $57.5 \%$ \\
11) Crampi & $4.1 \%$ & $28.8 \%$ & $67.1 \%$ \\
12) Disgeusia & $2.7 \%$ & $15.1 \%$ & $82.2 \%$ \\
13) Nausea & $0.0 \%$ & $15.1 \%$ & $84.9 \%$ \\
14) Vomito & $0.0 \%$ & $9.6 \%$ & $90.4 \%$ \\
\hline
\end{tabular}

TABELLA III - VARIABILI DIFFERENTI IN MODO STATISTICAMENTE SIGNIFICATIVO PER OGNI SINTOMO (SCORE 0 VS 1 VS 2)

\begin{tabular}{|c|c|c|c|c|c|c|}
\hline & & score 0 & score 1 & score 2 & $\mathrm{p}$ & \\
\hline \multirow[t]{2}{*}{ Sete } & Osm & $301 \pm 8$ & $306 \pm 9$ & $310 \pm 10$ & 0.030 & 0 vs 2 \\
\hline & BUN & $60.5 \pm 19.5$ & $71.8 \pm 15.5$ & $77.4 \pm 20.7$ & 0.037 & 0 vs 2 \\
\hline \multirow[t]{2}{*}{ Astenia } & Età & $52.0 \pm 17.4$ & $65.8(56.7-71.7)$ & $70.4 \pm 5.3$ & 0.013 & 0 vs 2 \\
\hline & Alb & $4.5 \pm 0.3$ & $4.3 \pm 0.3$ & $4.2(3.9-4.4)$ & 0.027 & 0 vs 2 \\
\hline Insonnia & $\mathrm{Ca}$ & $9.8(9.6-10.3)$ & $9.9(9.2-10.3)$ & $10.2(10.0-10.7)$ & 0.041 & $0-1$ vs 2 \\
\hline \multirow[t]{2}{*}{ Ipersonnia } & $\mathrm{pO}_{2}$ & $88.7 \pm 11.7$ & $83.7 \pm 12.1$ & $78.3 \pm 11.2$ & 0.033 & 0 vs 2 \\
\hline & $\mathrm{Na}$ & $136.7 \pm 3.0$ & $137.7 \pm 2.7$ & $139.0 \pm 2.4$ & 0.049 & 0 vs 2 \\
\hline Anoressia & K & $5.6 \pm 0.7$ & $5.4 \pm 0.8$ & $4.2(4.0-5.1)$ & 0.020 & 0 vs 2 \\
\hline \multirow[t]{3}{*}{ Dispnea } & Età & $64.1(54.6-68.3)$ & $70.9(63.3-74.8)$ & $72.7 \pm 2.2$ & 0.004 & 0 vs 2 \\
\hline & $\mathrm{Na}$ & $137.0 \pm 2.9$ & $137.7 \pm 2.8$ & $141.3 \pm 1.2$ & 0.029 & 0 vs 2 \\
\hline & $\mathrm{K}$ & $5.6 \pm 0.8$ & $5.4 \pm 0.8$ & $4.4 \pm 0.5$ & 0.049 & 0 vs 2 \\
\hline Disgeusia & $\mathrm{pO}_{2}$ & $89.3(78.3-96.5)$ & $79.0(73.7-83.4)$ & $69.2(65.9-72.5)$ & 0.017 & 0 vs 2 \\
\hline \multirow[t]{5}{*}{ Nausea } & $\mathrm{BE}$ & $-2.2 \pm 3.1$ & $+0.8 \pm 4.0$ & & 0.008 & 0 vs 1 \\
\hline & $\mathrm{pH}$ & $7.39 \pm 0.04$ & $7.43 \pm 0.04$ & & 0.009 & 0 vs 1 \\
\hline & $\mathrm{HCO}_{3}$ & $21.9 \pm 3.0$ & $24.6 \pm 4.2$ & & 0.018 & 0 vs 1 \\
\hline & $\mathrm{pO}_{2}$ & $86.5 \pm 11.6$ & $77.9 \pm 13.8$ & & 0.039 & 0 vs 1 \\
\hline & BUN & $74.8 \pm 18.5$ & $61.9 \pm 22.0$ & & 0.043 & 0 vs 1 \\
\hline \multirow[t]{3}{*}{ Vomito } & $\mathrm{BE}$ & $-2.1 \pm 3.2$ & $+2.5 \pm 2.4$ & & 0.003 & 0 vs 1 \\
\hline & $\mathrm{HCO}_{3}$ & $22.0 \pm 3.2$ & $26.4 \pm 2.2$ & & 0.003 & 0 vs 1 \\
\hline & $\mathrm{pH}$ & $7.39 \pm 0.04$ & $7.44 \pm 0.03$ & & 0.032 & 0 vs 1 \\
\hline
\end{tabular}

$\operatorname{cosi}(n=1)$. Le caratteristiche della popolazione sono riassunte in Tabella I. Tutti i pz sono dializzati 3 volte/settimana. La durata mediana delle sedute dialitiche è di $218^{\prime}$, range 165 '-240'. La bicarbonato-dialisi standard (bic-HD) è eseguita in 60 $\mathrm{pz}$ e l'emodiafiltrazione in 16 . I pz diabetici sono sei.

\section{Sintomi}

I pz sono stati interrogati una sola volta da un unico operatore Medico Nefrologo riguardo la presenza, a domicilio, di quattordici sintomi: astenia, anoressia, nausea, vomito, disgeusia, prurito, artralgie, crampi, restless legs syndrome (RLS), dispnea, insonnia, ipersonnia, sete, tremori. Per disgeusia si è inteso "difficoltà a distinguere i diversi sapori dei cibi", per artralgie "dolore alle spalle", per insonnia "risveglio notturno senza riaddormentamento", per ipersonnia la "sonnolenza prima di pranzo", per sete il "soffrire la sete". La presenza o assenza dei sintomi è stata quantificata secondo la frequenza con tre risposte possibili: "mai" (score 0), "non tutti i giorni" (score 1) e "tutti i giorni" (score 2).

\section{Variabili}

Contemporaneamente alla raccolta dei sintomi, i pz sono stati sottoposti ad un prelievo ematico dalla fistola arterovenosa e ad uno arterioso dall'arteria femorale per l'emogasanalisi (EGA) subito prima della seduta di metà settimana. L'EGA è stato eseguito su settanta $\mathrm{pz}$ poiché tre hanno posto rifiuto. Le variabili seriche studiate sono: sodio $(\mathrm{Na})$, potassio (K), BUN, creatinina ( $\mathrm{Cr})$, calcio $(\mathrm{Ca})$, fosforo $(\mathrm{P})$, paratormone intatto (PTHi), albumina (Alb), glucosio (Gluc), emoglobina $(\mathrm{Hb})$, $\mathrm{pH}$, bicarbonati $\left(\mathrm{HCO}_{3}\right), \mathrm{pO}_{2}, \mathrm{pCO}_{2}$, base excess $(\mathrm{BE})$ e osmolalità plasmatica (Osm). Le altre variabili analizzate sono state il $\mathrm{Kt} / \mathrm{V}$ calcolato con la formula di Daugirdas (3), l'età anagrafica in anni e quella dialitica in mesi.

\section{Analisi statistica}

Le variabili continue sono espresse come media \pm deviazione standard o 
come mediana (range interquantile) in caso di distribuzione non Gaussiana. L'ANOVA ad una via con il test post-hoc di Tukey-Kramer o il test di Kruscal-Wallis sono stati utilizzati per confrontare le variabili. L'ipotesi nulla è stata rifiutata per valori di $\alpha<0.05$ in tutti i test. L'analisi statistica è stata eseguita con il software JMP 3.02 (SAS Institute) su Apple Macintosh.

\section{Risultati}

La presenza e frequenza dei quattordici sintomi è riassunta in Tabella II. I risultati statisticamente significativi per ogni sintomo sono riassunti in Tabella III. Nausea e vomito non sono stati riferiti "tutti i giorni" (score 2) da alcun pz e l'analisi statistica è stata quindi condotta su 2 popolazioni (score 0 vs 1 ). RLS, tremori, crampi, artralgie e prurito non sono risultati in relazione con alcuna delle variabili studiate.

Sete: la sete è risultato il sintomo più frequente in assoluto ed è purtroppo costante nella metà dei nostri pz. È piuttosto evidente il ruolo della elevata BUN e Osm (Fig.1).

Astenia: dopo la sete è il sintomo più frequente ed il $25 \%$ dei nostri pz lo riferisce giornalmente. La prevalenza dello score $1(63.0 \%)$ depone per un effetto legato alle sedute dialitiche. L'effetto dell'età anagrafica elevata sembra decisivo nell'indurre tale sintomatologia: i pz con astenia persistente hanno tutti più di 63 anni.

Artralgie: le artralgie, definite come "dolori alle spalle", sono risultate il terzo sintomo più frequente coinvolgendo quasi l' $80 \%$ dei pz. Coloro i quali non riferiscono artralgie presentano un valore mediano di età dialitica di 3 anni e di PTHi di $95 \mathrm{ng} / \mathrm{mL}$.

Insonnia: il Ca è l'unico parametro risultato in diretta relazione con la frequenza di episodi di insonnia (Fig. 2). Tra i $15 \mathrm{pz}$ che riferiscono insonnia persistente ben $12(80 \%)$ presentano $\mathrm{Ca}>10 \mathrm{mg} / \mathrm{dL}$ nel predialisi con vero-

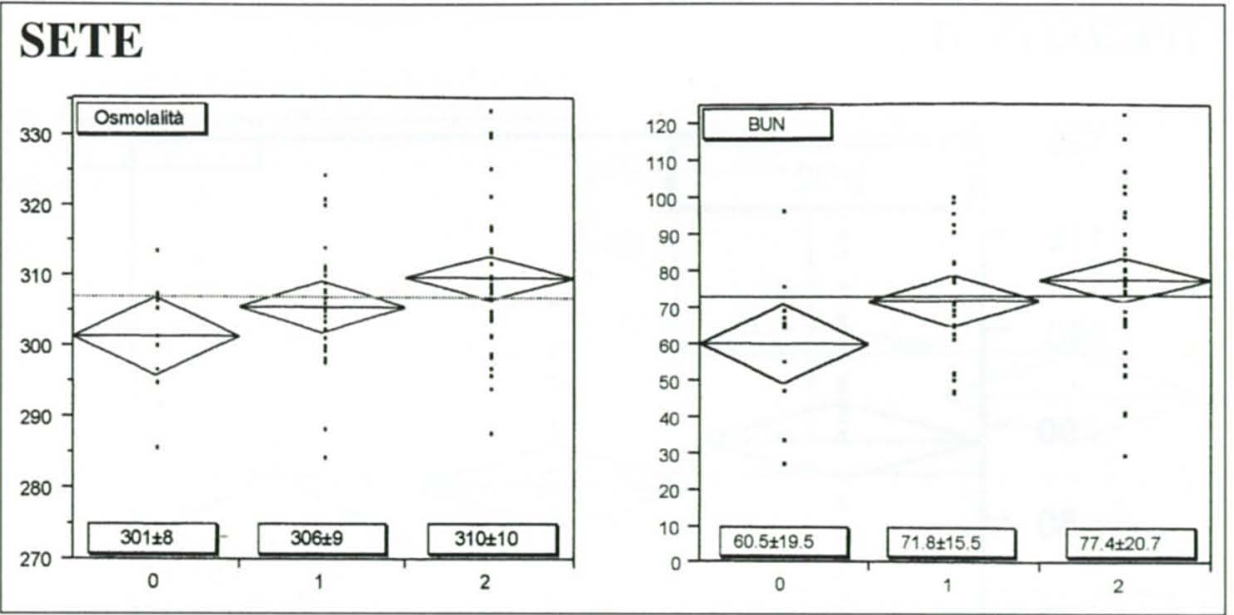

Fig. 1 - Valori medi di osmolalità e BUN nei pazienti emodializzati con sete assente (score=0), saltuaria (score $=1$ ) e giornaliera (score $=2$ ).

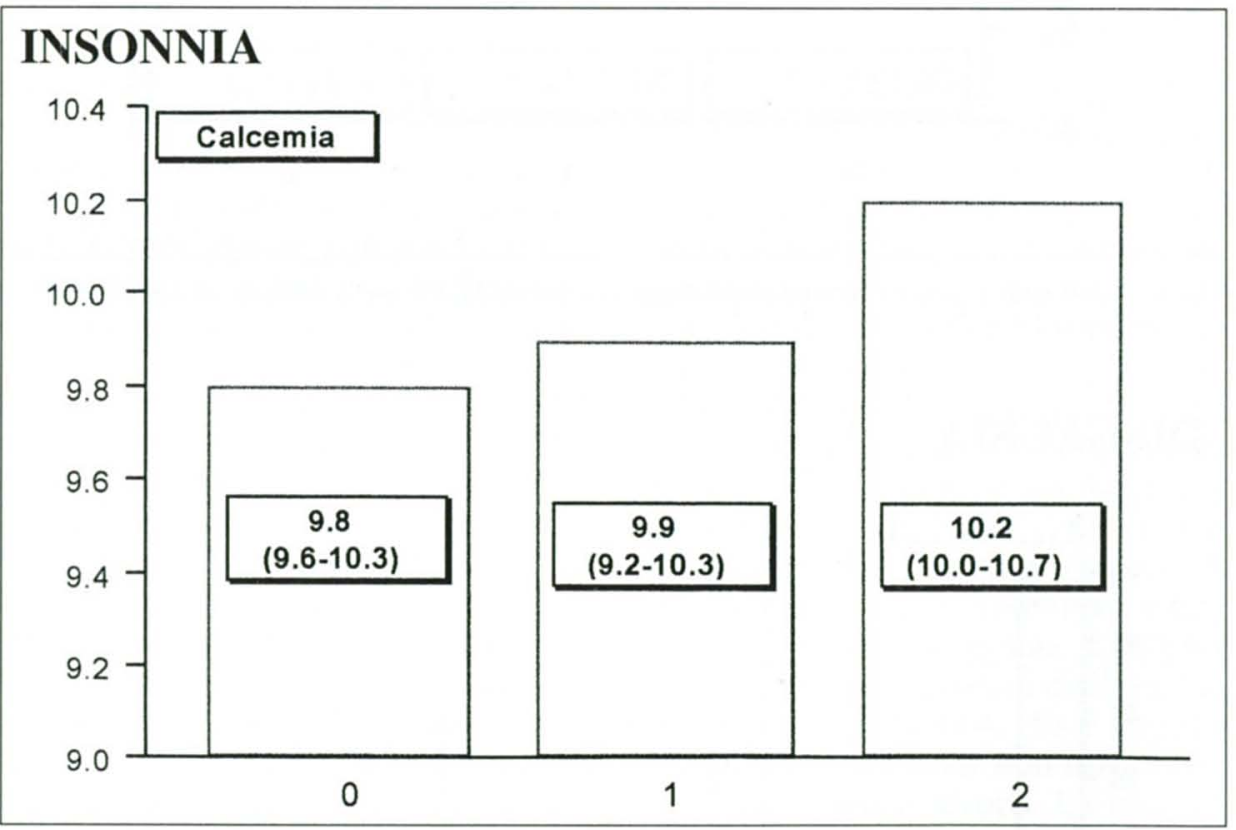

Fig. 2 - Valori mediani di calcemia nei pazienti emodializzati con insonnia assente (score = 0), saltuaria (sco$r e=1)$ e giornaliera ( score $=2$ ).

simile discreta ipercalcemia nella maggior parte del periodo interdialitico.

Ipersonnia: la $\mathrm{pO}_{2}$ ridotta è risultata in grado di influenzare la comparsa di ipersonnia (Fig. 3) che sembra risentire anche del Na per valori crescenti.

Prurito: solo il 33\% dei nostri pz è risultato completamente libero dal prurito ed è più spesso incostante (score $1=57.5 \%$ ) (Tab. II) suggerendo un legame con le sedute dialitiche.
Anoressia: più della metà dei nostri $\mathrm{pz}$ riferisce assenza totale di anoressia e solo l' $8 \%$ la accusa in modo costante. I valori di $\mathrm{K}$ sono risultati in relazione inversa alla frequenza di anoressia. Ben $5 / 6 \mathrm{pz}$ con anoressia= 2 presentavano $\mathrm{K}<4.7 \mathrm{mEq} / \mathrm{L}$ e $\mathrm{BUN}<66 \mathrm{mg} / \mathrm{dL}$.

RLS: l'incidenza di RLS nel nostro studio è risultata modesta $(8.2 \%$ persistente) con assenza totale nel $75 \%$ dei nostri pz. 


\section{IPERSONNIA}

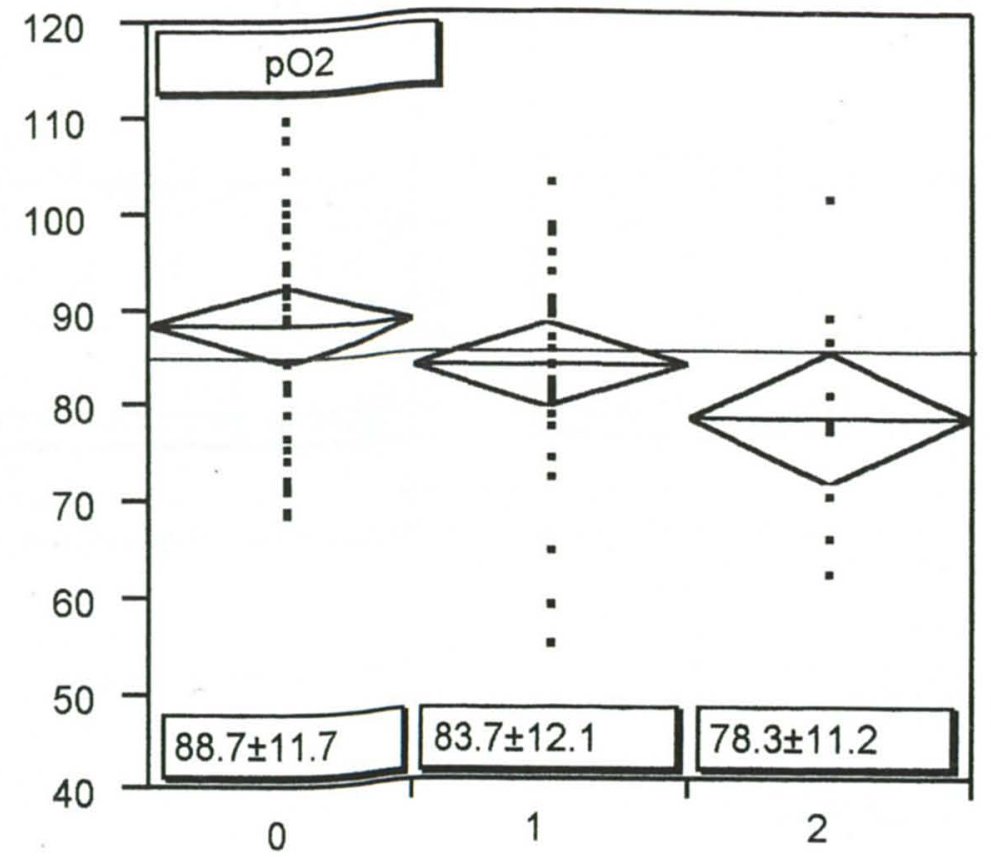

Fig. 3 - Valori medi di $\mathrm{pO}_{2}$ nei pazienti emodializzati con ipersonnia assente (score $=0$ ), saltuaria $($ score $=1)$ e giornaliera $($ score $=2$ ).

\section{DISGEUSIA}

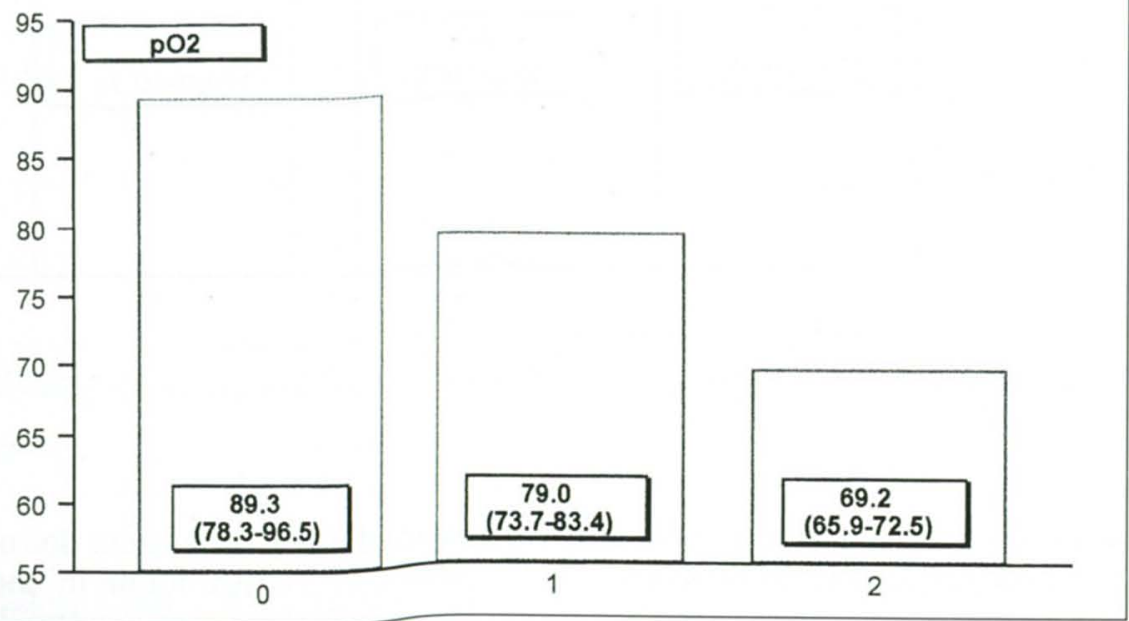

Fig. 4 - Valori medi di $\mathrm{O}_{2}$ nei pazienti emodializzati con disgeusia assente (score $\left.=0\right)$, saltuaria $($ score $=1) e$ giornaliera ( score $=2$ ).

Tremori: il tremore è un sintomo poco frequente con il $75 \%$ dei $\mathrm{pz}$ che non lo riferisce affatto.

Dispnea: anche se meno della metà dei $\mathrm{pz}$ accusa dispnea saltuaria o costante per l'importanza soggettiva del sintomo tale frequenza non va considerata trascurabile. Elevata età anagrafica, ipernatremia e ipopotassiemia sono risultati in relazione con la dispnea.

Crampi: ben $1 / 3$ dei pz accusa crampi a domicilio che costituisce un disturbo non trascurabile.

Disgeusia: dai nostri dati, la disgeusia appare un sintomo infrequente essendo assente in più dell' $80 \%$ dei pz. La $\mathrm{pO}_{2}$ per valori decrescenti è l'unica variabile risultata significativa con la frequenza di disgeusia (Fig. 4). La $\mathrm{pO}_{2}$ è risultata mediamente $<80 \mathrm{e}$ $<70 \mathrm{mmHg}$ nei gruppi con score $1 \mathrm{e}$ 2 rispettivamente.

Nausea: la nausea insieme al vomito è il sintomo più raramente riferito con 1 ' $85 \%$ dei pz completamente libero e nessuno con riscontro giornaliero. Gli 11 soggetti con nausea hanno tutti un $\mathrm{pH}>7.390$ con ipossiemia secondaria ad alcalosi metabolica (Fig. 5). I sei pz diabetici non riferiscono nausea e non presentano alcalosi metabolica (BE mediano -2.6 con range -5.1/-0.7). La nausea sembra piuttosto indipendente dal vomito poiché solo $3 / 11 \mathrm{pz}$ con nausea riferiscono anche vomito. Nessun pz con età $<57$ anni riferisce nausea.

Vomito: il vomito è il sintomo più raro, riferito, solo saltuariamente, dal $10 \%$ circa dei pz. Solo $3 / 7$ pz con vomito riferiscono anche nausea. Piuttosto sorprendente il riscontro di assenza totale del vomito nei $6 \mathrm{pz}$ diabetici, tutti però con $\mathrm{BE}<0$ (vedi sopra). E presente una chiara relazione tra vomito ed alcalosi metabolica ed è significativo che tutti i pz con vomito, così come per la nausea, hanno un $\mathrm{pH}>7.390$ in predialisi.

\section{Discussione}

Sete: l'effetto dipsogenico della BUN è stato dimostrato nei ratti (4). L'Osm efficace è il risultato dei valori plasmatici di Na e Gluc ma in uremia anche gli elevati valori di BUN potrebbero influenzare l'Osm spiegando la significatività di entrambi i parametri nel nostro studio. In HD, tra le molecole che possiamo considerare osmoticamente attive (Na, Gluc, BUN) solo la BUN è presente in concentrazioni abnormemente elevate. 


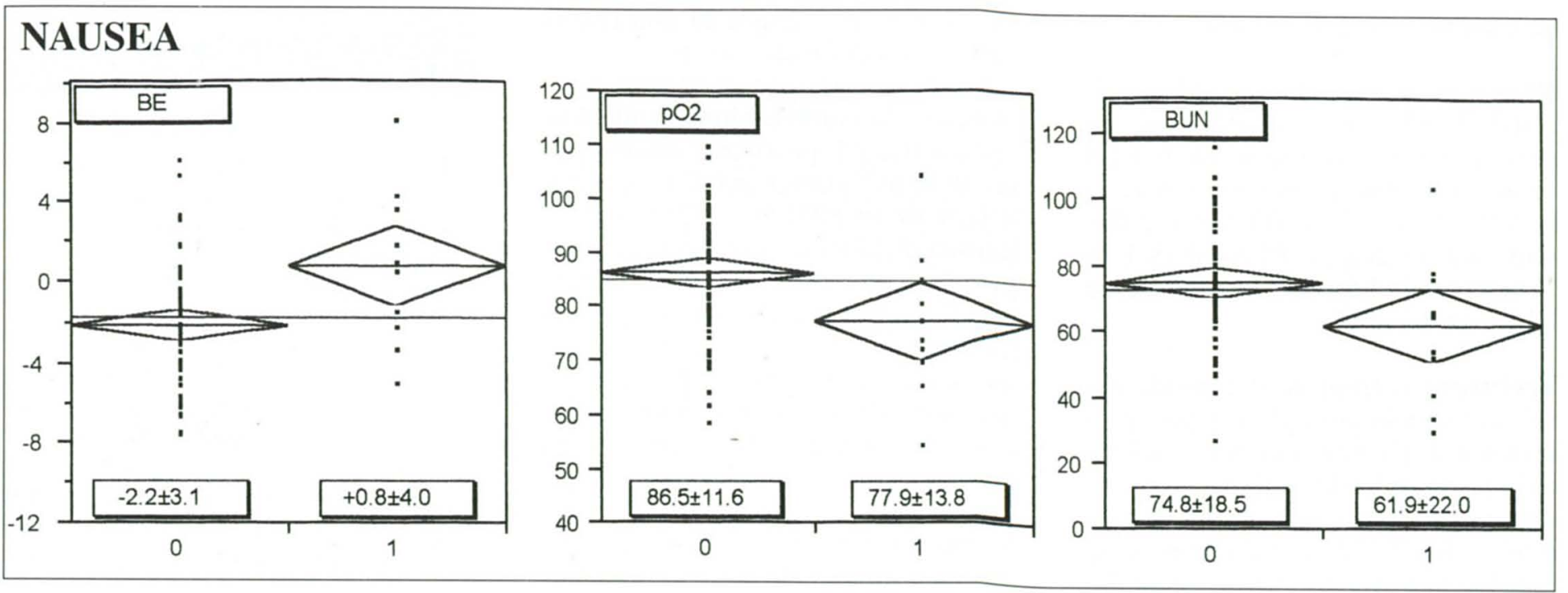

Fig.5 - Valori medi di BE, $\mathrm{pO}_{2}$ e BUN nei pazienti emodializzati con nausea assente (score $\left.=0\right)$ e saltuaria $($ score $=1)$.

Astenia: l'effetto dell'elevata età anagrafica sulla presenza di astenia è evidente ed il sintomo è riferito soprattutto dopo la seduta dialitica. L'ipoalbuminemia nei $\mathrm{pz}$ con più frequente astenia è di difficile interpretazione anche se potrebbe rappresentare un epifenomeno legato alla senilità dei pz più astenici.

Artralgie: l'influenza della età dialitica sull'insorgenza di artralgie è apparsa evidente a 20 anni dall'introduzione della dialisi cronica come conseguenza dei depositi di un tipo di amiloide denominata $\beta 2$-amiloide per l'identificazione della B2microglobulina come agente precursore (5). L'effetto della età dialitica nel nostro modello statistico non è risultato significativo perché nel gruppo con score 2 è mediamente vicina ai 12 anni, molto più elevata rispetto ai 3 e 1.5 anni dei gruppi con score 0 e 1 ma l'andamento di questi valori, $3-1.5-12$, non è crescente. È possibile che due differenti patologie, come la 32 -amiloidosi e l'iperparatiroidismo secondario agiscano in età dialitiche differenti: la prima, molto tardivamente, nella seconda decade di trattamento sostitutivo e la seconda in qualsiasi momento della storia dialitica; l'interferenza delle due condizioni potrebbe essere sufficiente ad influenzare la nostra analisi statistica.
Insonnia: 1'ipercalcemia, in grado di indurre, se severa, astenia, anoressia, nausea, vomito e confusione mentale, è risultata in relazione all'insonnia e questa associazione non risulta precedentemente segnalata. In HD un dializzante con Ca ionizzato di 1.25 $\mathrm{mmol} / \mathrm{L}(2.5 \mathrm{mEq} / \mathrm{L})$ è in grado di indurre un bilancio calcico positivo durante la seduta mentre con 0.75 $\mathrm{mmol} / \mathrm{L}$ il bilancio è sicuramente negativo (6). Nel nostro Centro, il $\mathrm{Ca}$ nel dialisato è $1.75 \mathrm{mmol} / \mathrm{L}$ per bic$\mathrm{HD}, \mathrm{BF}$ e $\mathrm{PFD}$ e $2.0 \mathrm{mmol} / \mathrm{L}$ in AFB. Una dimostrata riduzione dell'insonnia contemporanea a quella della concentrazione di $\mathrm{Ca}$ nel dializzante potrà confermare l'ipotesi di questo legame causale.

Ipersonnia: considerando l'ipersonnia come un sintomo neurologico il riscontro di un effetto negativo dell' $i$ possiemia non sorprende perché già dimostrato sulla neuropatia (7) mentre il significato della relativa ipersodiemia è di difficile interpretazione.

Prurito: nessuno parametro è risultato in relazione statisticamente significativa con il prurito ed in particolare il PTHi, prima imputato della genesi del prurito $(8,9)$ e poi assolto (10). La teoria dell'alterato metabolismo degli ioni divalenti, cioè l'ipercalcemia e l'iperfosforemia (8-10), non viene confermata. Nel nostro studio, il prurito è riferito più frequentemente come incostante. Questo dato, insieme all'assenza di relazione con i parametri analizzati, conferma la possibilità che la sua etiologia sia legata a fenomeni biochimici con insorgenza nell'ambito della seduta dialitica. Con questa ipotesi concordano le teorie secondo le quali il rilascio di istamina (11), l'attivazione delle citochine mediata dal contatto sangue-membrana (12) e neurotrasmettitori liberati durante la seduta dialitica (13) sarebbero coinvolti nella sua insorgenza. L'effetto positivo dell'adeguatezza dialitica sul prurito descritta $(14,15)$ e negata $(2,16)$ in diversi studi non trova conferma nel nostro lavoro. La precisa etiologia del prurito in HD rimane oscura.

Anoressia: l'anoressia nel pz uremico è stata messa in relazione all'uremia per se', a fattori psicologici, economici e sociali, ai farmaci, alla gastropatia, alla nausea e al vomito. Nel nostro studio, i valori ridotti di $\mathrm{K}$ nei $\mathrm{pz}$ con anoressia sono probabilmente l'effetto (ridotto introito) e non la causa del sintomo. La presenza di anoressia sembra sottostimata e i $7 / 11 \mathrm{pz}$ con nausea che non dichiarano anoressia supportano tale ipotesi. La combinazione di ridotti valori di K e BUN può essere considerata una spia di importante mancanza di appe- 
tito, anche se non dichiarata.

RLS: nessuna variabile studiata è risultata in relazione statisticamente significativa con la frequenza di RLS in accordo con quanto già riportato in letteratura (17). L'Hb non è differente nei tre gruppi (RLS $=0$ vs 1 vs 2) smentendo l'ipotetica associazione RLS-anemia (18).

Tremore: il tremore è considerato un sintomo neurologico e può presentarsi in pz non uremici se affetti dal morbo di Parkinson, da ipertiroidismo, in terapia con antidepressivi o metoclopramide e in condizione di ipoglicemia. La frequente secondarietà del sintomo, insieme alla sua scarsa frequenza e all'assenza di relazioni con le variabili pongono dubbi sul suo rapporto elettivo con l'uremia.

Dispnea: l'importanza dell'età anagrafica per la dispnea non sorprende, mentre il significato dei valori di $\mathrm{K}$ decrescenti e la sicura ipopotassiemia post-dialitica dei $\mathrm{pz}$ con score 2 $(\mathrm{K}<5.0 \mathrm{mEq} / \mathrm{L})$ suggerisce un ruolo della debolezza muscolare che potrebbe coinvolgere anche i muscoli respiratori. La relativa ipersodiemia dei pz dispnoici suggerisce una possibile relazione tra $\mathrm{Na}$ e suo pool corporeo.

Crampi: i crampi sono un sintomo che è stato studiato soprattutto durante la seduta emodialitica ma è riferito anche a domicilio, soprattutto di notte. L'assenza di relazioni statistiche con i parametri studiati non ci permette di elaborare un approccio preventivo.

Disgeusia: le alterazioni del gusto nei $\mathrm{pz}$ in HD possono essere secondarie anche all'assunzione di farmaci come gli ACE-inibitori e gli antiistaminici e sono messe in relazione anche ad un deficit di zinco (19) ma non vi sono in letteratura dati riguardo la relazione con l'ipossiemia da noi verificata. L'effetto negativo dell'età anagrafica avanzata, descritto in letteratura (20), non ha trovato riscontro nel nostro studio e la relazio- ne tra uremia e disgeusia non risulta tuttora ben definita.

Nausea: la combinazione di alcalosi metabolica ed ipossiemia sembra poter indurre l'insorgenza di nausea nei nostri pz in HD. Solo 3/11 pz con nausea dichiarano anche vomito e ciò può fare escludere ragionevolmente che l'alcalosi metabolica dei pz con nausea sia secondaria al vomito. La nausea dei nostri emodializzati sembra coinvolgere l'aspetto nutrizionale con riduzione della BUN (Fig. 5) da verosimile minore intake proteico.

Vomito: il vomito è risultato il sintomo meno frequente ed è sorprendentemente assente nei nostri pz diabetici che costituiscono una popolazione a rischio (21). L'alcalosi metabolica sembra essere la causa più che la conseguenza del vomito poiché anche i pz con nausea presentano la stessa relazione con l'elevato BE.

\section{Conclusioni}

In conclusione, $\mathrm{i} \mathrm{pz}$ in HD sono affetti da una importante sintomatologia. I dati del nostro studio suggeriscono che in HD un attento e periodico monitoraggio dell'equilibrio acido-base arterioso e della ionemia sono importanti, soprattutto nei pz anziani, al fine di ridurre, almeno in parte, la sintomatologia e migliorare, quindi, la loro qualità della vita. La personalizzazione, con periodiche modifiche, della composizione del dializzante riguardo a $\mathrm{NaHCO}_{3}, \mathrm{~K}$ e $\mathrm{Ca}$ appare una strategia razionale per ottenere tali obiettivi. L'importanza dell' adeguatezza dialitica in HD, se espressa in modo tradizionale con indici come il $\mathrm{Kt} / \mathrm{V}$, risulta ridimensionata e tale concetto andrebbe allargato verso un'espressione di adeguata terapia sostitutiva della funzione omeostatica renale.

\section{BIBLIOGRAFIA}

1. Steuer RR, Leypoldt JK, Cheung AK, Senekjian HO, Conis JM. Reducing symptoms during hemodialysis by continuously monitoring the hematocrit. Am J Kidney Dis 1996; 27 (4):525-32.

2. Brunet $P$, Saingra Y, Leonetti F, Vacher-Coponat H, Ramananarivo $\mathrm{P}$, Berland $\mathrm{Y}$. Tolerance of haemodialysis: a randomized cross-over trial of 5-h versus 4-h treatment time. Nephrol Dial Transplant 1996; 11(Suppl 8):4651.

3. Daugirdas JT. The post/pre plasma urea nitrogen ratio to estimate $\mathrm{Kt} / \mathrm{V}$ and NPC: mathematical modeling. Int J Artif Organs 1989; 12:411-9.

4. Giovannetti S, Barsotti G, Cupisti A. Dipsogenic effect of urea in rats. Nephron 1993; 64 (4): 587-91.

5. Gejyo F, Odani S, Yamada T, Homma N, Saito H, Suzuki Y, Nakagawa Y, Kobayashi H, Maruyama $Y$, Hirasawa $Y$, Suzuki M, Arakawa M. B2-microglobulin: a new form of amyloid protein associated with chronic hemodialysis. Kidney Int 1986; 30:385-90.

6. Hou SH, Zhao J, Ellman CF, $\mathrm{Hu} J$, Griffin Z, Spiegel DM, Bourdeau JE. Calcium and phosphorus fluxes during hemodialysis with low calcium dialysate. Am J Kidney Dis 1991; 18:21724.

7. Stewart AG, Waterhouse JC, Howard P. Cardiovascular autonomic nerve function in patients with hypoxaemic chronic obstructive pulmonary disease. Eur Respir J 1991; 4:1207.

8. Massry SG, Popovtzer MM, Coburn JW, Makoff DL, Maxwell MH, Kleeman CR. Intractable pruritus as a manifestation of secondary hyperparathyroidism in uremia: disappearance of itching 
after subtotal parathyroidectomy. N Engl J Med 1968; 279:697-700.

9. Hampers CL, Katz AI, Wilson RE, Merrill JP. Disappearance of "uremic" itching after subtotal parathyroidectomy. N Engl J Med 1968; 279:695-7.

10. Carmichael AJ, McHugh MM, Martin AM, Farrow M. Serological markers of renal itch in patients receiving long term hemodialysis. Br Med J 1988; 296 : 1575 .

11. Stockenhuber F, Kurz RW, Sertl K, Grimm G, Balcke P. Increased plasma histamine levels in uraemic pruritus. Clin Sci 1990; 79:477-82.

12. Cheung AK, Parker CJ, Wil$\operatorname{cox}$ L, Janatova J. Activation of the alternative pathway of complement by cellulosic hemodialysis membranes. Kidney Int 1989; $36: 257-65$.

13. Kessler M, Moneret-Vautrin DA, Cao-Huu T, Mariot A, Chanliau J. Dialysis pruritus and sensitization (letter). Nephron 1992; 60:241.

14. Masi CM, Cohen EP. Dialysis efficacy and itching in renal failure. Nephron 1992; 62:257-61.

15: Hiroshige K, Kabashima N, Takasugi M, Kuroiwa A. Optimal dialysis improves uremic pruritus. Am J Kidney Dis 1995; 25 (3):413-9.

16. Carmichael AJ, McHugh MI, Martin AM. Itch unrelated to adequacy of haemodialysis (letter). Br Med J 1989; 297:95.

17. Winkelman JW, Chertow GM, Lazarus JM. Restless legs syndrome in end-stage renal disease. Am J Kidney Dis 1993; 28 (3):372-8.

18. Roger SD, Harris DCH, Stewart JH. Possible relation between restless legs and anemia in renal dialysis patients. Lancet $1991 ; 1: 1551$.

19. Burge JC, Park HS, Whi- tlock CP, Schemmel RA. Taste acuity in patients undergoing long-term hemodialysis. Kidney Int 1979; 15:49-53.

20. Fernström A, Hylander B, Rössner S. Taste acuity in patients with chronic renal failure. Clin Nephrol 1996; 45(3):169-74.

21. Eisengerg B, Murata GH, Tzamaloukas AH, Zager PG, Avasthi PS. Gastroparesis in diabetics on chronic dialysis: clinical and laboratory associations and predictive features. Nephron 1995; 70:296-300. 